\title{
SYSTOLIC ARRAYS FOR MULTIDIMENSIONAL DISCRETE TRANSFORMS
}

\author{
Weicheng Shen \\ Martin Marietta Laboratories \\ Baltimore, MD 21227-3848 \\ A. Yavuz Oruç \\ Electrical Engineering Department \\ University of Maryland \\ College Park, MD 20740 \\ Submitted in April 1989 \\ Revised in April 1990
}

\begin{abstract}
An active area of research in supercomputing is concerned with mapping certain finite sums, such as discrete Fourier transforms, onto arrays of processors. This paper presents systolic mapping techniques that exploit the parallelism inherent in discrete Fourier transforms. It is established that, for an M-dimensional signal, parallel executions of such transforms are closely related to mappings of an $(\mathrm{M}+1)$-dimensional finite vector space into itself. Three examples of such parallel schemes are then described for the discrete Fourier transform of a two-dimensional finite extent sequence of size $N_{1} \times N_{2}$. The first is a linear array of $N_{1}+N_{2}-1$ processors and takes $O\left(N_{1} N_{2}\right)$ steps. The second is an $N_{1} \times N_{2}$ rectangular array of processors and takes $O\left(N_{1}+N_{2}\right)$ steps, and the third is a hexagonal array which uses $N_{1} N_{2}+\left(N_{2}-1\right)\left(N_{1}+N_{2}-1\right)$ processors and takes $O\left(N_{1}+N_{2}\right)$ steps. All three mappings are optimal in that they achieve asymptotically highest speedup possible over the sequential execution of the same transform, and can easily be generalized to higher dimensions.
\end{abstract}




\section{Introduction}

Certain finite sums such as discrete Fourier transforms (DFTs) find extensive applications in many fields such as signal processing [Blahut 1985, Dudgeon and Mesereau 1984, Oppenheim and Shafer 1984] pattern recognition [Blahut 1985, Jacobson and Wechsler 1984], computer vision [Gafni and Zeevi 1979], computer arithmetic [Ling and Bayoumi 1988], and tomography [Hinshaw and Lent 1983]. In most of these fields, one often deals with very large sets of discrete signals which require more sophisticated techniques than conventional sequential transformation schemes. For example, the fastest sequential DFT algorithm takes $O(N \log N)$ steps on a onedimensional, $N$-point array which should be iterated $M$ times for a $M$-dimensional array, leading to $O\left(M N^{M} \log N\right)$ time complexity. While this order of complexity may be acceptable for small values of $N$ and $M$, certain design constraints such as the sampling rate and bandwidth of input signals, often make it unacceptable to carry out discrete transforms on multidimensional signals by sequential means.

For faster DFT computations, a number of parallel architectures have been proposed in the literature [Cappello and Steiglitz 1983, Chakrabarti and Ja'Ja' 1988, Chowdary et al. 1984, Gertner and Shamash 1987, Miranker and Winker 1984, Zhang and Yun 1984]. [Thompson 83] examined the VLSI complexity of one-dimensional DFTs using the area $\times \operatorname{time}^{2}\left(A T^{2}\right)$ model and surveyed several one-dimensional DFT algorithms. [Zhang and Yun 1984] presented a meshconnected processor array implementation of a two-dimensional DFT algorithm. [Chowdary et al. 1984] described a fast two-dimensional DFT processor. Recently, [Gertner and Shamash 1987] introduced a hybrid architecture approach based on the well-known row-column decomposition algorithm. They described two hybrid architectures, each using a butterfly array of processors to perform a one-dimensional DFT and an interconnection network to reroute the intermediate results back to the array between different phases of the algorithm. One of these architectures uses a perfect shuffle network [Stone 1971] while the other uses what is called a rotation network to align the outputs of the processor array between different phases of the computation. For an $N_{1} \times N_{2} \times \ldots \times N_{M}$ array, where $N_{1} N_{2} \ldots N_{M}=N$, the architecture with the rotation network achieves $A T^{2}=\left(N^{2} \log ^{2} N\right)$ which is optimal. More recently, [Chakrabarti and Ja'Ja' 1988] generalized the rotation network concept to obtain $A T^{2}$ optimal designs, but with other space and time tradeoffs.

While the designs in [Chakrabarti and Ja'Ja' 1988, Gertner and Shamash 1987] meet the $A T^{2}$ lower bound for computing linear transforms, they require rotation networks with $O\left(N_{1}^{2} N_{2}^{2} \ldots N_{M}^{2}\right)$ area for an $N_{1} N_{2} \ldots N_{M}$ array of inputs. Moreover, these networks are difficult to layout in VLSI [Franklin 1981]. To avoid the interconnection problems associated with these designs, this paper examines three systolic implementations of the row-column decomposition algorithm within the 
context of a program graph contraction procedure which was described in [Shen and Oruç 1989]. Such implementations have been described for other algorithms in the literature [Leiserson 1981, Kung and Leiserson 1980]. The systolic arrays given in this paper extend these implementations to the row-column decomposition algorithm by using simple last-in/first-out (LIFO) memories for the alignment of intermediate results. For an $N_{1} \times N_{2}$ data array, the first implementation uses a linear array of $N_{1}+N_{2}-1$ processors and takes $O\left(N_{1} N_{2}\right)$ steps. The second implementation employs an $N_{1} \times N_{2}$ rectangular array of processors and takes $O\left(N_{1}+N_{2}\right)$ steps, and the third one uses a hexagonal array of $N_{1} N_{2}+\left(N_{2}-1\right)\left(N_{1}+N_{2}-1\right)$ processors and takes $O\left(N_{1}+N_{2}\right)$ steps. All three have asymptotically optimal speedup when compared with the sequential implementation of the row-column decomposition algorithm. Moreover, these implementations directly generalize to $M$-dimensional signals without sacrificing their optimality. It is also shown that these systolic implementations are just three instances of a wide spectrum of optimal systolic contactions of the row-column decomposition algorithm.

The rest of the paper is organized as follows. Section 2 describes the program graph model and the contraction mapping procedure. Section 3 describes the program graph for the row-column decomposition algorithm. In Section 4, the mapping procedure of Section 2 is used to obtain systolic contractions of this program graph. Section 5 discusses the memory organization and interconnections of the resulting processor arrays. In Section 6, the contractions of Section 4 are generalized to discrete transformations of finite extent multidimensional signals. The paper is concluded in Section 7.

\section{Approach}

The processor arrays to be described subsequently are all derived from systolic contractions of the row-column decomposition algorithm [Dudgeon and Mersereau 1984]. Before we describe these, it is first necessary to outline the main elements of our contraction procedure.

The procedure begins with a labelled program graph representation of the row-column decomposition algorithm. Such representations, which have been used in the literature [Cytron 1988, Ramakrishnan et al. 1986, Ramakrishnan and Varman 1984] earlier, are captured by a directed acyclic graph $G=\langle V, E, D>$, where (1) $V$ is a set of vertices, each representing a computation step (computation vertex), an external input (source vertex), or a result from computation (sink vertex); (2) $E$ is a set of directed edges which specify the dependences between the computation vertices in $V$; and (3) $D$ is a set of delays assigned to the edges in $E$ so that the delay $d$ associated with an edge $(a, b) \in E$ indicates that the operation at vertex $b$ is to be initiated precisely $d$ clock ticks after the initiation of the operation at vertex $a$. A program graph is mapped to a processor array by partitioning its computation vertices into disjoint subsets and assigning each 


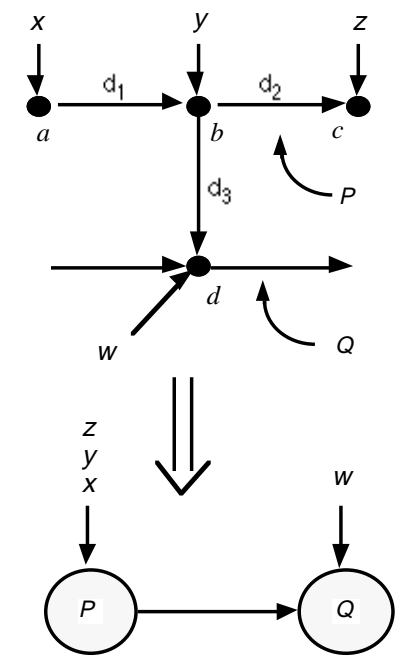

Figure 1. Contraction of a program graph.

subset to a separate processor. Depending on the partition, this results in a processor array with a particular topology which will be called a target array or a systolic contraction of that program graph. Computations in each group of vertices are then carried out by the processor, to which they are assigned, in a sequential order and at a uniform rate as regulated by a global clock. The interconnections between processors are determined as follows. Whenever two computation vertices are merged, all the edges between the two are deleted, and all incoming and outgoing edges to these two vertices are reconnected to the resultant vertex without altering their orientations. The duplicate edges between the resultant vertices are also deleted unless their orientations are different. The delays to be assigned to the links between the processors in the target array are determined by algebraically summing them to zero around each loop of oriented edges much like the voltages of branches within a loop add up to zero in an electrical circuit. As we shall see subsequently, satisfying these delay equations simultaneously leads to processor arrays with very simple processing elements. The determined delay values are used to schedule the arrivals of the inputs. The exact locations at which the inputs enter a target array are determined in part by the manner in which the computation vertices are partitioned and assigned to the processors in the target array. A more formal presentation of the mapping procedure is given in [Shen 1987, Shen and Oruç 1989]; the following example provides a brief description.

Figure 1 depicts a program graph with four computation vertices, $a, b, c, d$. Partitioning them into sets $\{a, b, c\}$ and $\{d\}$ results in the target array with two processors $P$ and $Q$, also shown in the figure. The computation vertices $a, b$, and $c$ are assigned to processor $P$, and computation vertex 
$d$ is assigned to processor $Q$. These assignments dictate that inputs $x, y$, and $z$ enter processor $P$ in the order shown and with delay $d_{1}$ between $x$ and $y$, and delay $d_{2}$ between $y$ and $z$. On the other hand, input $w$ enters processor $Q d_{3}$ clock ticks after $y$ enters $P$ to allow the required time for the transmission of the data from processor $P$ to processor $Q$ along the link that connects the two. Thus, a key constraint in assigning the computation vertices to processors and scheduling the inputs for entry is that the processors fire their computations only when they have all of their inputs available. Furthermore, processors have no decision capability, implying that they repeatedly execute the same computation without knowing what their inputs are. All they know is that they must compute whatever they see at their inputs at predetermined beats of time, for example, $t=1$ second., $t=3$ seconds, $t=5$ seconds and so on. When a program graph does not contain any loops of oriented edges, this constraint can easily be enforced by assigning each link the minimum delay it can tolerate. When it does, we assign the delays to the links by summing the delays to zero around each loop of oriented edges and simultaneously satisfying the resulting equations.

In the following sections this procedure will be used to synthesize three systolic contractions of the row-column decomposition algorithm.

\section{The Row-column Decomposition Algorithm}

In its most general form, the DFT of an $M$-dimensional sequence, $x\left(n_{1}, n_{2} \ldots\right.$, $\left.n_{M}\right)$, is expressed as [Dudgeon and Mersereau 1984]

$$
y\left(k_{1}, k_{2}, \ldots k_{M}\right)=\sum_{n_{1}, n_{2}, \ldots, n_{M}} x\left(n_{1}, n_{2}, \ldots, n_{M}\right) W_{N_{1}}^{n_{1} k_{1}} W_{N_{2}}^{n_{2} k_{2}} \ldots W_{N_{M}}^{n_{M} k_{M}}
$$

where indicies $n_{i}, k_{i}$ run over the domain $\left[0, N_{i}-1\right], 1 \leq i \leq M$ and $W_{N_{i}}^{n_{i} k_{i}}=\exp \left(-j \frac{2 \pi n_{i} k_{i}}{N_{i}}\right)$ are complex exponentials.

Several fast algorithms are known for efficiently computing this weighted sum. Among them, the row and column decomposition algorithm is highly modular, that is, one can compute the DFT of an $M$-dimensional signal by carrying it out $M$ times over the indices $n_{M}, n_{M-1}, \ldots, n_{2}, n_{1}$ in an iterative manner. For example, if $M=2$, then

$$
y\left(k_{1}, k_{2}\right)=\sum_{0 \leq n_{1} \leq N_{1}-1} \sum_{0 \leq n_{2} \leq N_{2}-1} x\left(n_{1}, n_{2}\right) W_{N_{1}}^{n_{2} k_{2}} W_{N_{2}}^{n_{1} k_{1}},
$$

or if we let

$$
G\left(n_{1}, k_{2}\right)=\sum_{0 \leq n_{2} \leq N_{2}-1} x\left(n_{1}, n_{2}\right) W_{N_{2}}^{n_{2} k_{2}}
$$

then

$$
y\left(k_{1}, k_{2}\right)=\sum_{0 \leq n_{1} \leq N_{1}-1} G\left(n_{1}, k_{2}\right) W_{N_{1}}^{n_{1} k_{1}} .
$$


Thus we can compute $y\left(k_{1}, k_{2}\right)$ in two steps: First compute Equation (3), then compute Equation (4). Although both sums are computable, they are not in the best form to evaluate since we need to simplify the weights $W_{N_{1}}^{n_{1} k_{1}}$ and $W_{N_{2}}^{n_{2} k_{2}}$, that is, compute $n_{1} k_{1} \bmod N_{1}$ and $n_{2} k_{2} \bmod N_{2}$ when $n_{1} k_{1} \geq N_{1}$, and $n_{2} k_{2} \geq N_{2}$. This problem can be avoided if we use Horner's rule of summation and rewrite these sums recursively as given below:

\section{Initial Step:}

$G^{(0)}\left(n_{1}, k_{2}\right)=0,0 \leq n_{1} \leq N_{1}-1,0 \leq k_{2} \leq N_{2}-1 ;$

$y^{(0)}\left(k_{1}, k_{2}\right)=0,0 \leq k_{1} \leq N_{1}-1,0 \leq k_{2} \leq N_{2}-1$.

\section{Recursive Steps:}

Phase 1: (Compute $G\left(n_{1}, k_{2}\right)$ )

for $n_{2}=0$ to $N_{2}-1$ do

$G^{\left(n_{2}+1\right)}\left(n_{1}, k_{2}\right):=G^{\left(n_{2}\right)}\left(n_{1}, k_{2}\right) W_{N_{2}}^{n_{2} k_{2}}+x\left(n_{1}, N_{2}-n_{2}-1\right)$.

Phase 2: (Compute $y\left(k_{1}, k_{2}\right)$ )

for $n_{1}=0$ to $N_{1}-1$ do

$y^{\left(n_{1}+1\right)}\left(k_{1}, k_{2}\right):=y^{\left(n_{1}\right)}\left(k_{1}, k_{2}\right) W_{N_{1}}^{n_{1} k_{1}}+G\left(N_{1}-n_{1}-1, k_{2}\right)$.

Notice that we now need only the weights $W_{N_{i}}^{k_{i}} ; 0 \leq k_{i} \leq N_{i}-1,1 \leq i \leq 2$ to carry out the algorithm. It will be assumed that these weights are precomputed and stored in a memory device. This way, they can be accessed directly without any address computation since we need not compute $n_{1} k_{1} \bmod N_{1}$ and $n_{2} k_{2} \bmod N_{2}$ anymore. This assumption is not unreasonable given that a storage device with $O\left(N_{1}+N_{2}\right)$ words should suffice, and that the original problem size, $N_{1} N_{2}$, is an order of magnitude greater than $O\left(N_{1}+N_{2}\right)$. In view of this assumption, the row-column decomposition algorithm for a two-dimensional DFT requires $N_{1} N_{2}^{2}$ multiply-add operations for the first phase, and $N_{1}^{2} N_{2}$ for the second phase, a total of $N_{1} N_{2}\left(N_{1}+N_{2}\right)$ steps.

These formulas generalize to $M$ dimensions in a straighforward way; the DFT of a $N_{1} \times N_{2} \times \ldots \times N_{M}$ sample can be computed using the row-column decomposition algorithm in $N_{1} N_{2} \ldots N_{M}\left(N_{1}+N_{2}+\right.$ $\left.\ldots+N_{M}\right)$ multiply-add steps. If $N_{1}=N_{2}=\ldots=N_{M}=N$, then the total number of multiply-add operations becomes $M N^{M+1}$. It should be noted that, from Equation (1), the direct computation of an $M$-dimensional DFT needs $(M+1) N^{2 M}$ steps ( $N^{M}$ additions and $M N^{M}$ multiplications for each of the $N^{M}$ instances of the $M$-tuple $\left.k_{1} k_{2} \ldots k_{M}\right)$. Thus, the row-column decomposition 
method reduces the total number of steps by a factor of $O\left(N^{M-1}\right)$, which is considerable. However, this is still too slow for large values of $M$, and we need to resort to parallel schemes.

The parallel schemes we shall describe in subsequent sections are based on systolic processor arrays [Kung and Leiserson 1980]. To map the row-column decomposition algorithm on a systolic array, we first represent each phase of the algorithm by a program graph, as shown in Figure 2 . In Figure 2(a), vertical and oblique lines represent inputs $x\left(n_{1}, n_{2}\right), 0 \leq n_{1} \leq N_{1}-1,0 \leq n_{2} \leq N_{2}-1$, and complex exponentials $W_{N_{2}}^{k_{2}}, 0 \leq k_{2} \leq N_{2}-1$, respectively. Horizontal lines represent partial sums, $G\left(n_{1}, k_{2}\right)$, which will be used as an input sequence in the next phase of computation. Each computation vertex multiplies its horizontal and oblique inputs, adds this product to its vertical input, and sends the result along the horizontal edge to the next vertex. Thus $G^{\left(n_{2}+1\right)}\left(n_{1}, k_{2}\right):=$ $G^{\left(n_{2}\right)}\left(n_{1}, k_{2}\right) W_{N_{2}}^{k_{2}}+x\left(n_{1}, n_{2}\right)$ is evaluated along the horizontal path of $N_{2}$ computation vertices $\left(N_{2}=3\right.$ in Figure 2(a)), which starts with source vertex $G^{0}\left(n_{1}, k_{2}\right)$ and ends with destination vertex $G^{N_{2}}\left(n_{1}, k_{2}\right)$.

Figure 2(b) shows the program graph for the second phase of the algorithm. In this case, the vertical and oblique lines represent intermediate results $G\left(n_{1}, k_{2}\right), 0 \leq n_{1} \leq N_{1}-1,0 \leq k_{2} \leq N_{2}-1$, and complex exponentials $W_{N_{1}}^{k_{1}}, 0 \leq k_{1} \leq N_{1}-1$, respectively. The horizontal lines represent the final sums $y\left(k_{1}, k_{2}\right)$, which are the desired results. Observe that the program graphs in Figure 2(a) and 2(b) each represent a one-dimensional DFT. In general, the DFT of an $M$-dimensional input can be represented by $M$ program graphs where the first graph describes the one-dimensional DFT of the input along the first axis, the second graph describes the one-dimensional DFT along the second axis, and so on.

\section{Row-column Decomposition on Systolic Arrays}

This section describes how to map the program graph given in Section 3 on three systolic arrays with linear, rectangular, and hexagonal geometries. In each case, we will establish that the given design asymptotically achieves the highest speed up possible, and therefore, is asymptotically optimal. These designs will later be extended to $M$-dimensional transforms in Section 6.

\section{Linear Array Implementation}

First, consider the linear array implementation. By starting out with the program representation of the row-column decomposition algorithm, we can obtain a linear processor array by grouping the computation vertices which fall on the vertical lines as shown in Figure 3. For an $N_{1} \times N_{2}$ sequence, this results in a linear array with $N_{1}+N_{2}-1$ processors as illustrated in Figure 4 for $N_{1}=N_{2}=3$. The processors are labelled $P_{i}, 0 \leq i \leq N_{1}+N_{2}-2$ where $P_{0}$ denotes the rightmost processor and $P_{N_{1}+N_{2}-2}$ denotes the leftmost processor. 

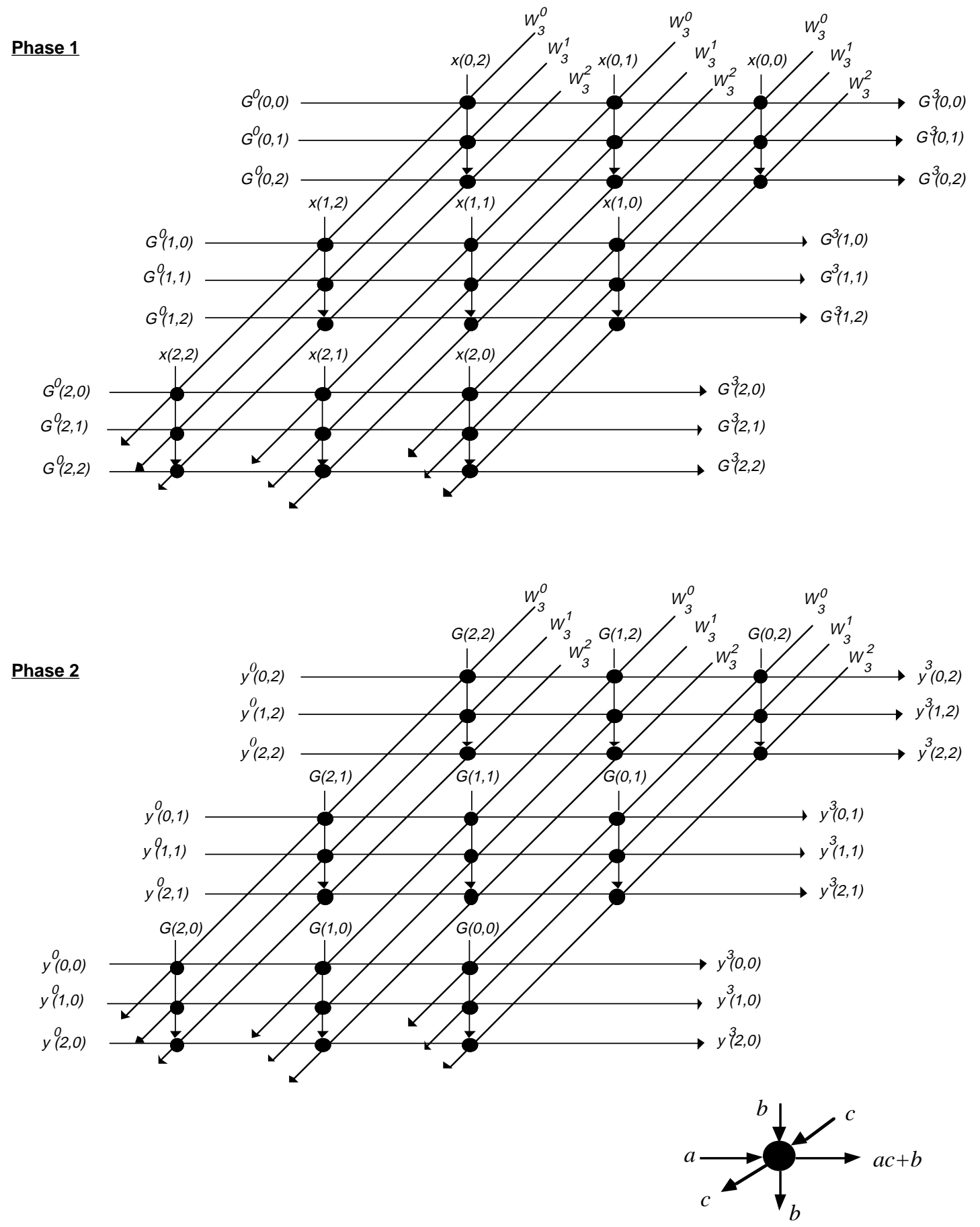

Computation at each vertex

Figure 2. A program graph for the row-column decomposition algorithm. 



Figure 3. A contraction which results in a linear array.

The row-column algorithm is carried out by this array in two phases. In the first phase, all inputs $x(i, j)$ for which $i+j=k$ are fed into processor $P_{k}$ in ascending row order, that is, the entries with the smallest row index first, the entries with the next smallest row index next, and so on. This is in keeping with the order among $x(i, j)$ as established by the time paths of Figure 3(a). Moreover, 

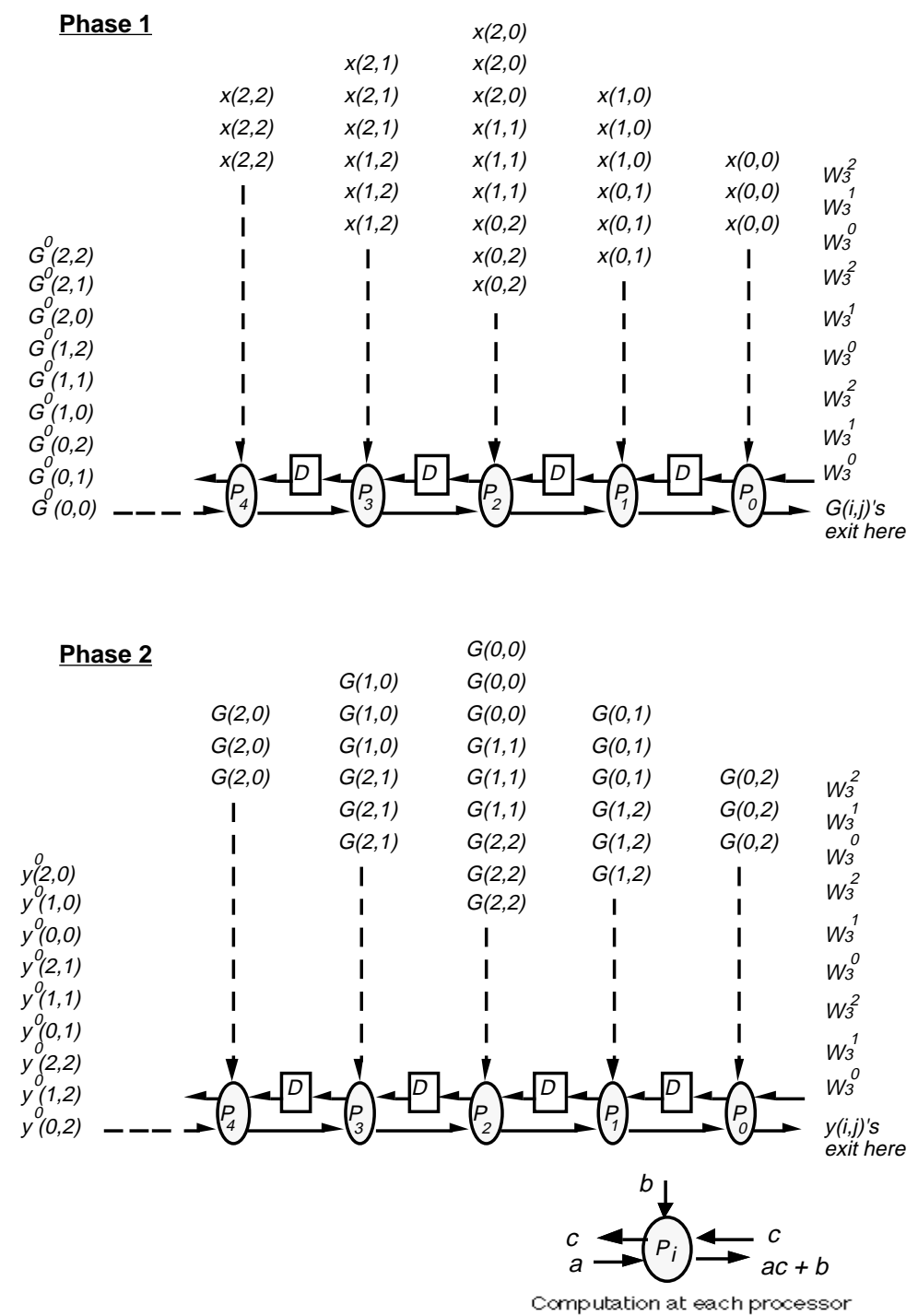

Figure 4. Row-column decomposition on a linear array.

each $x(i, j)$ is fed into the array $N_{2}$ times since each processor needs to execute $N_{2}$ computation vertices per each group of horizontal lines in the original program.

As a second group of entries, the weights $W_{N_{2}}^{i}, 0 \leq i \leq N_{1}-1$, enter the array through $P_{0}$ and proceed to the left at the same speed as $x(i, j)$ but with $N_{2}-1$ times the delay between consecutive 
entries in $x$ (twice for the processor array of Figure 4). The elements of $G$ enter the array at processor $P_{N_{1}+N_{2}-1}$ and march to the right one clock tick at a time. The increase in the delays between consecutive entries in the weight sequence is necessary for the correct timing of the computations at the processors and is accomplished by the delay elements inserted between consecutive processors. This can be seen by examining a loop of delays shown in heavy lines in Figure 3. As stated in Section 2, for the target array to be systolic, the algebraic sum of the delays in every loop of edges in the corresponding program graph must be zero. Using this fact we can write for the loop shown in Figure 3(a)

$$
d_{1}+d_{2}+d_{3}-d_{4}-d_{5}=0 .
$$

Furthermore, we let $d_{1}=d_{2}=d_{3}=1$ in order to make the delays between the consecutive entries in the $x$ sequence equal. This then leads to

$$
3-d_{4}-d_{5}=0
$$

which we can use to adjust the delays between the elements in the $G(i, j)$ and $W_{N_{2}}^{n_{2}}$ sequences. In the linear array of Figure 4 , we let $d_{4}=1$ and $d_{5}=2$ although it is possible to have other values for $d_{4}$ and $d_{5}$ as long as this equation is satisfied. Also, note that for an $N_{1} \times N_{2}$ input sequence, Equation (6) generalizes to $N_{2}-1-d_{4}-d_{5}=0$.

The arrivals of the inputs at the processors in Figure 4 are scheduled to meet the delays determined above. The elements of $x$ are skewed as they enter the processors. Similarly, the elements of $G$ are also skewed $N_{1}$ clock ticks as they enter the array through processor $P_{N_{1}+N_{2}-1}$. The computations in the second phase proceed similarly.

As for the time complexity, the total number of clock ticks to complete the first phase can be computed directly from the arrivals of the inputs at the processor in the middle, that is, processor $P_{N_{2}-1}$. It takes $N_{1}+N_{2}-1$ clock ticks for the first input to arrive and thereafter $N_{1} N_{2}$ clock ticks for the last input to arrive at this processor. At this time, the last element in the $G$ sequence also arrives at $P_{N_{2}-1}$ and needs $N_{2}$ clock ticks to exit from the rightmost processor. Adding these together, we have $N_{1}+2 N_{2}-1+N_{1} N_{2}$ clock ticks.

The second phase of the algorithm is identical to the first one, except that the $G$ sequence assumes the role of $x$, and $y$ replaces $G$ as output. The total time $T_{\text {linear }}$ to compute both phases of the row-column decomposition algorithm on a linear array with $N_{1}+N_{2}-1$ processors then becomes

$$
T_{\text {linear }}=2\left(N_{1} N_{2}+2 N_{1}+N_{2}-1\right) .
$$


Let $S_{\text {linear }}$ denote the speedup of the linear array over the sequential implementation of the row-column decomposition algorithm. Since it takes $N_{1} N_{2}\left(N_{1}+N_{2}\right)$ steps to perform the same algorithm on a single processor, we have

$$
S_{\text {linear }}=\frac{N_{1} N_{2}\left(N_{1}+N_{2}\right)}{2\left(N_{1} N_{2}+2 N_{1}+N_{2}-1\right)} .
$$

If $N_{1}=N_{2}=N$, then

$$
T_{\text {linear }}=2 N^{2}+6 N-2
$$

and

$$
S_{\text {linear }}=\frac{2 N^{3}}{2 N^{2}+6 N-2}=O(N) .
$$

This is asymptotically optimal since a total of $2 N-1$ processors is used.

The linear array described above is one of many arrays which can be obtained from the program graph of Figure 2. As another possibility, suppose that we assign all the $N_{1} N_{2}$ computation vertices within each horizontal block in Figure 2(a) to the same processor. This can easily be seen to lead to a linear array with $N_{1}$ processors. Likewise, by grouping the computation vertices in each horizontal block in Figure 2(b), we obtain a linear array with $N_{2}$ processors. Similarly, when all computation vertices in each diagonal block are assigned to the same processor, another linear array may be obtained. A detailed discussion of these and other linear array implementations will be deferred to another place. It suffices to say that these two implementations also result in a linear speedup as in the linear array given in Figure 4.

\section{Rectangular Array Implementation}

While the linear speedup is the best possible for a linear array implementation of the rowcolumn decomposition algorithm, it only reduces the total computation time to $O\left(N_{1} N_{2}\right)$ from $O\left(N_{1} N_{2}\left(N_{1}+N_{2}\right)\right)$. More can be done by using arrays with $O\left(N_{1} N_{2}\right)$ processors. To form such an array, we must partition the computation vertices in the program graph in Figure 2 into roughly $O\left(N_{1} \times N_{2}\right)$ processors. One possibility is as shown by the heavy lines in Figure 5 for $N_{1}=N_{2}=3$. In this case, each of the two phases of the algorithm is contracted along the antidiagonal paths to obtain the rectangular arrays of processors shown in Figure 6 . The number of processors is $N_{2} \times N_{2}$ for the first phase and $N_{1} \times N_{1}$ for the second phase. When $N_{1}=N_{2}=N$, both phases need $N^{2}$ processors. As for the assignment of computation vertices to the processors, all computation vertices on the $i$-th antidiagonal from the top left-hand corner where $0 \leq i \leq N_{1} N_{2}-1$ are assigned to the processor in row $1+\left\lfloor i / N_{1}\right\rfloor$ and column $i \bmod N_{2}$ in the target array. As can be seen from Figure 6, only the entries of $x$ and $G$ matrices move in the first phase and similarly only the entries of $y$ and $G$ move in the second phase. The weights are stored in registers local 

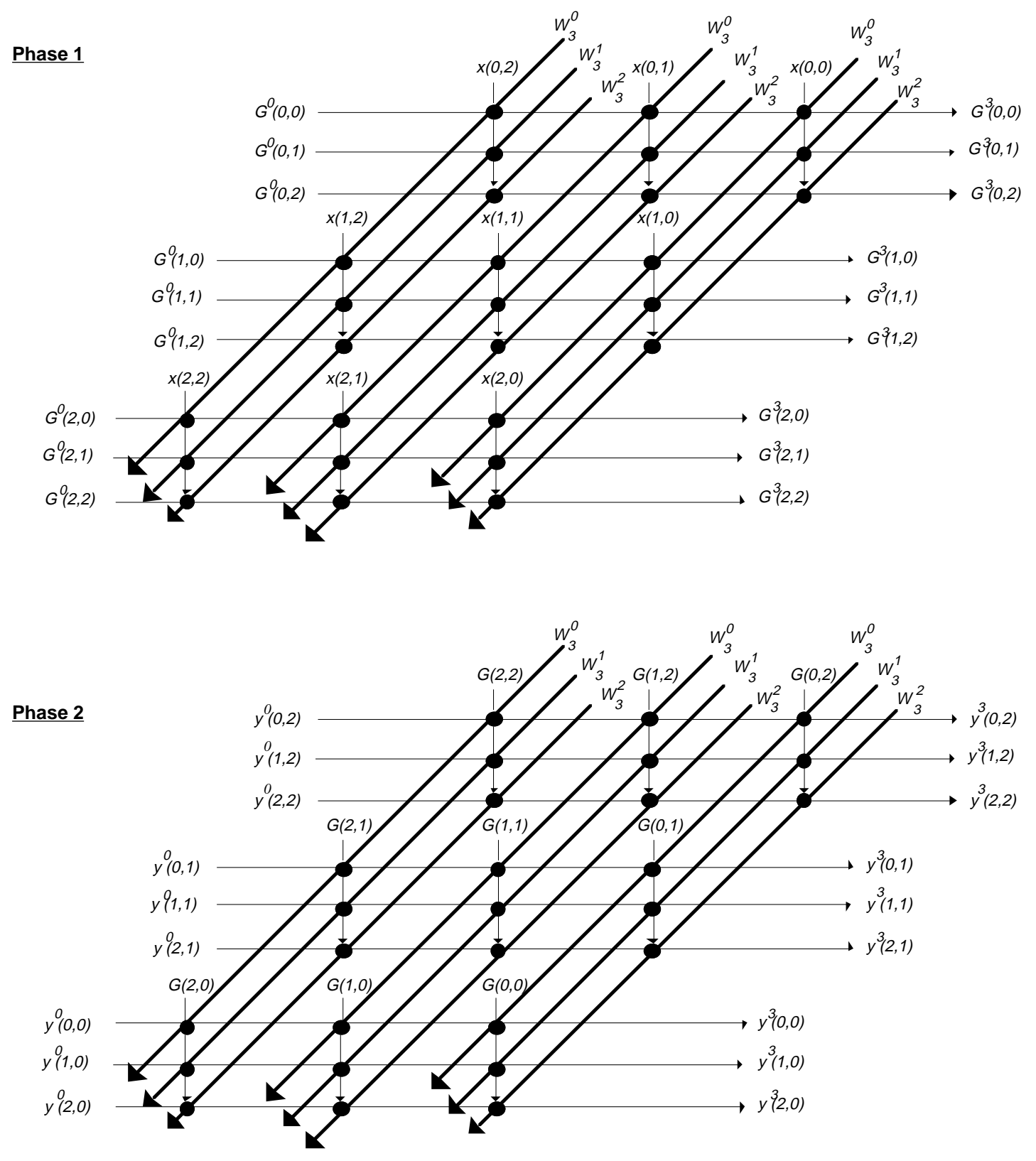

Figure 5. A contraction which results in a rectangular array.

to the processors in both phases. Also from the figure, it is obvious that the delays between the elements of $x$ must be the same as the delays between the elements of $G$ for the first phase, and similarly, the delays between the elements of $y$ and those between the entries of $G$ must be the same for the second phase. As for the arrivals of $x$ and $G$ matrices at the inputs of the array, it 


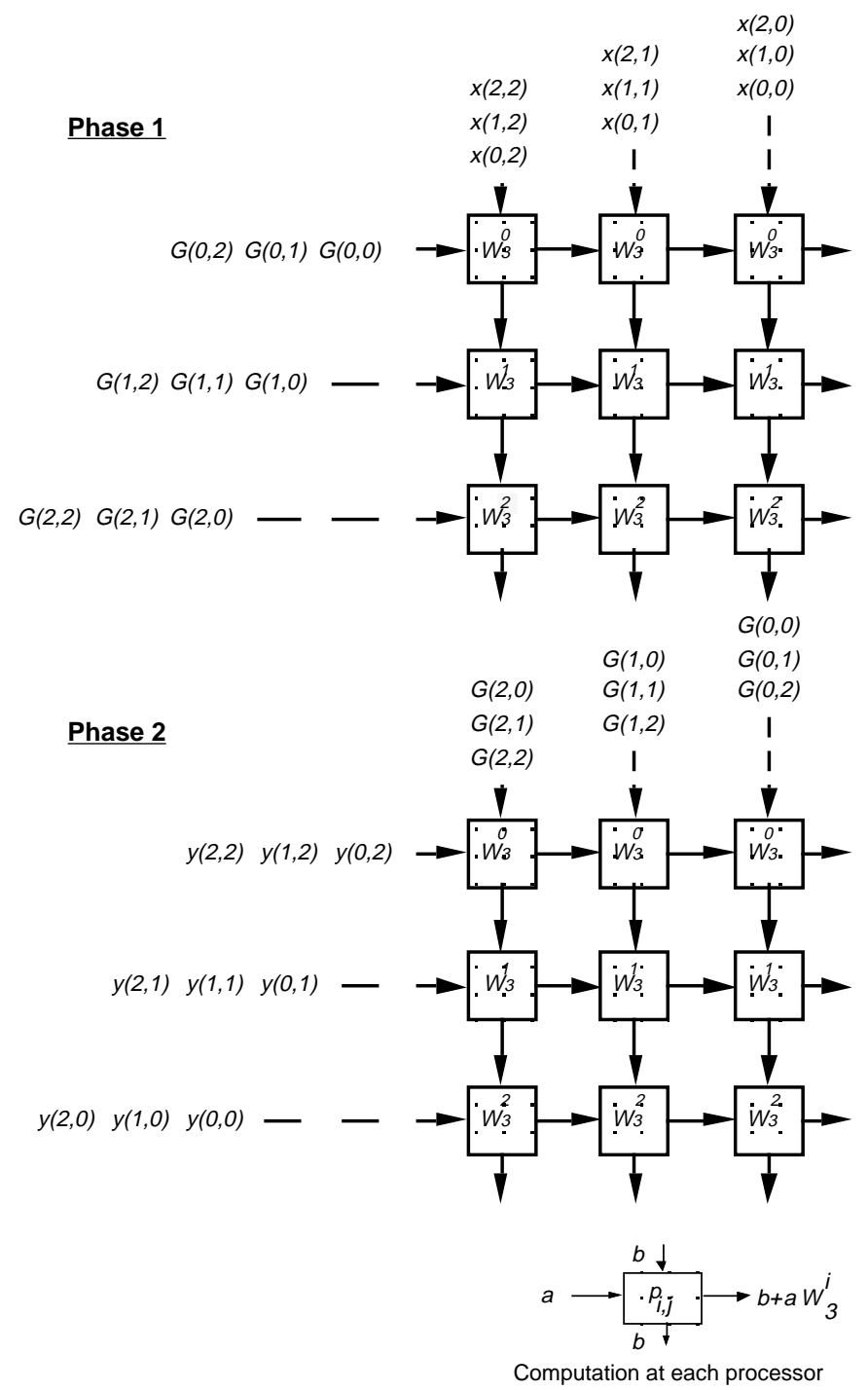

Figure 6. Row-column decomposition on a rectangular array.

is easily seen that they need to be skewed as shown in Figure 6 to achieve the correct timing of computations at the processors.

With these facts, it takes $2 N_{1}-1+N_{2}$ steps to complete the first phase, and $2 N_{2}-1+N_{1}$ steps 
to complete the second phase, and hence

$$
T_{\text {rectangular }}=3\left(N_{1}+N_{2}\right)-2
$$

steps to run the overall algorithm on an $N_{2} \times N_{2}$ rectangular array of processors for the first phase and an $N_{1} \times N_{1}$ rectangular array of processors for the second phase. It follows that the speedup over the single processor implementation is

$$
S_{\text {rectangular }}=\frac{N_{1} N_{2}\left(N_{1}+N_{2}\right)}{3\left(N_{1}+N_{2}\right)-2} .
$$

If we assume $N_{1}=N_{2}=N$, then

$$
T_{\text {rectangular }}=6 N-2
$$

and

$$
S_{\text {rectangular }}=\frac{2 N^{3}}{6 N-2}=O\left(N^{2}\right) .
$$

This again is asymptotically optimal as in the linear processor array case since a total of $N^{2}$ processors is used.

Another rectangular array implementation can be obtained by mapping each row of computation vertices to a different processor. In this case the horizontal inputs, -entries of $G$ matrix in the first phase, and those of $y$ matrix in the second phase- must be made stationary and stored in local memories of the processors. This, obviously, doubles the memory space needed by the earlier solution when $N_{1}=N_{2}=N$, since the earlier solution needs to store only the weights $W_{N}^{i, j}$ for both phases of the algorithm.

\section{Hexagonal Array Implementation}

To implement the row-column decomposition algorithm on a hexagonal array of processors, we partition the computation vertices as shown in Figure 7 for $N_{1}=N_{2}=3$. The resulting graph can then be contracted into a processor arrray (see Figure 8). It should be obvious that after the contraction, each processor vertex ends up having six distinct adjacent neighbors and thus can be modelled as a hexagonal cell. It can also be verified that the target array contains $N_{1} N_{2}+\left(N_{2}-1\right)\left(N_{1}+N_{2}-1\right)$ processors for the first phase and $N_{1} N_{2}+\left(N_{1}-1\right)\left(N_{1}+N_{2}-1\right)$ for the second phase. When $N_{1}=N_{2}=N$, both processor counts reduce to $3 N^{2}-3 N+1$.

Now, to describe which computation vertex is mapped to which processor, consider the $\alpha$ th column of computation vertices where $0 \leq \alpha \leq N_{1}+N_{2}-1$ in either graph in Figure 7. These can be partitioned into some $\beta N_{2} \times 1$ column matrices where $0 \leq \beta \leq N_{1}-1$. Let these column matrices be denoted by $C_{k}, 0 \leq k \leq \beta-1$ where $C_{1}$ is the top most matrix, $C_{2}$ is the next topmost matrix and 

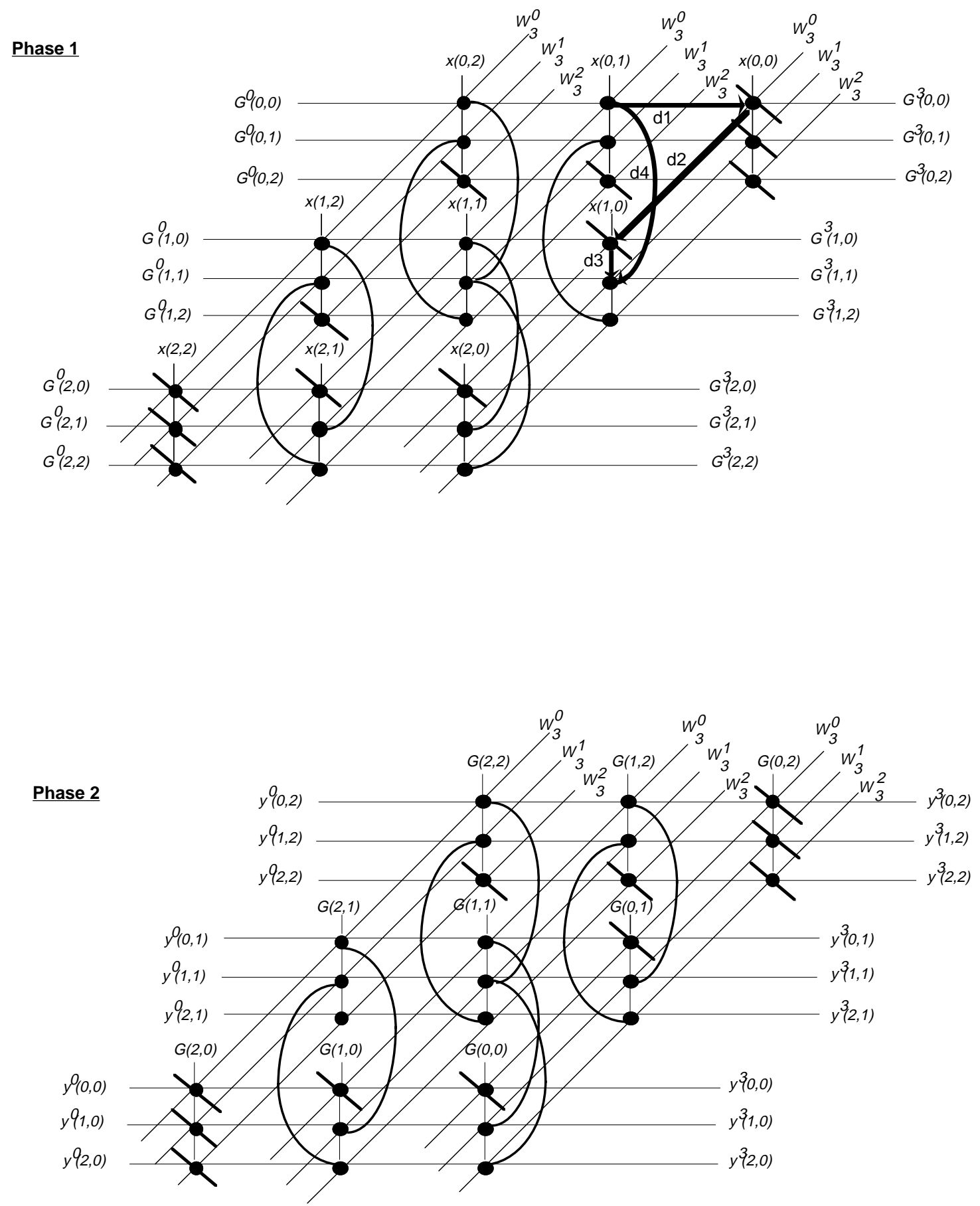

Figure 7. A contraction that results in an hexagonal array. 


\section{Phase 1}
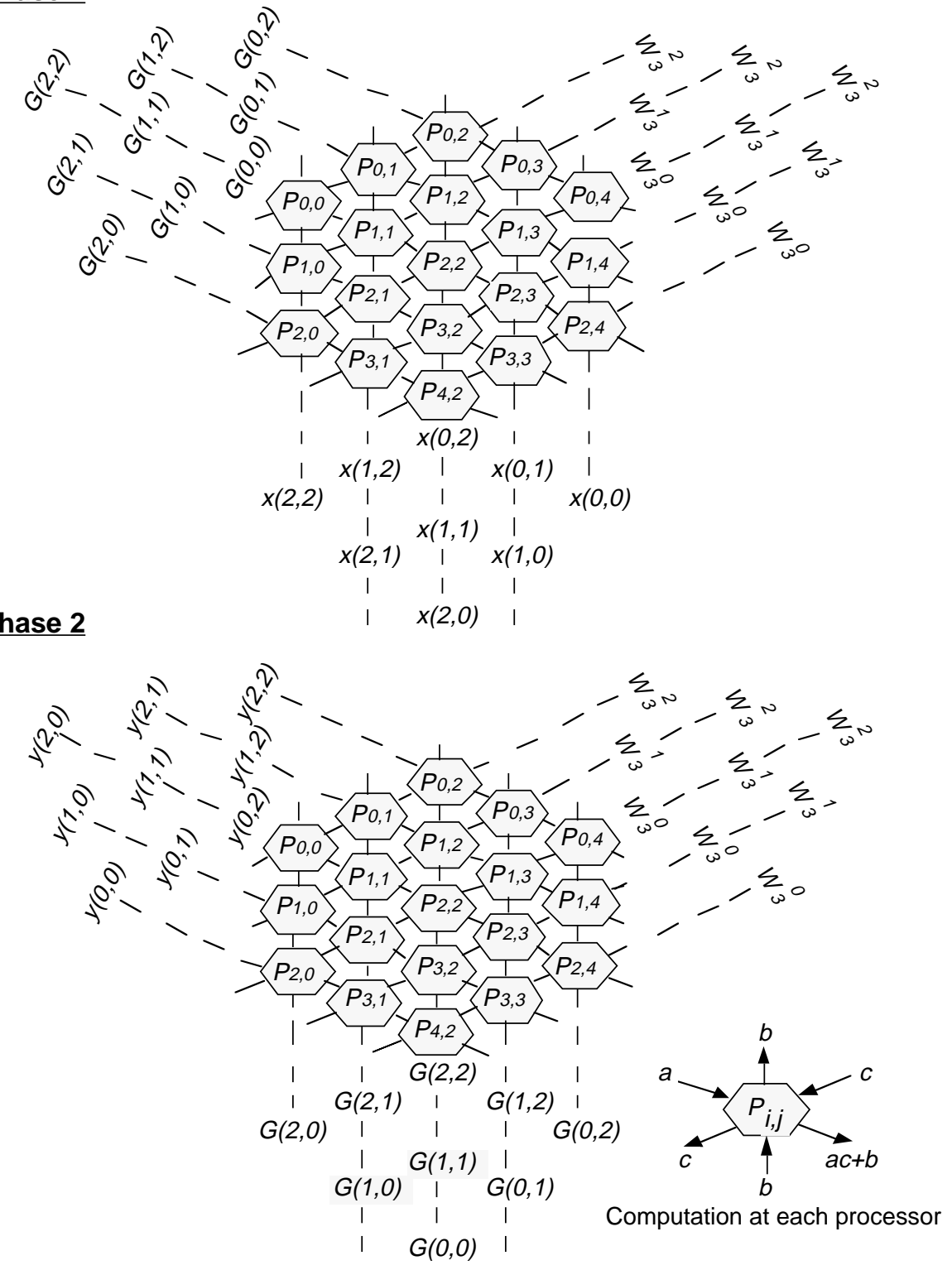

Figure 8. Row-column decomposition on a hexagonal array.

so on. Let $C=\left[C_{1}^{T} C_{2}^{T} \ldots C_{\alpha}^{T}\right]$. Then all computation vertices on each antidiagonal of $C$ are mapped to the same processor in the target array. More specifically, let $P_{0, \alpha}, P_{1, \alpha}, \ldots, P_{\beta+N_{2}-1, \alpha}$ denote the processor vertices in the $\alpha$ th column of the target array, where $P_{0, \alpha}$ is the topmost processor vertex, $P_{1, \alpha}$ is the next topmost processor vertex, and so on. Then all computation vertices on 
the $i$ th antidiagonal of $C$ from the top righthand corner are assigned to $P_{i, \alpha}, 0 \leq i \leq \beta+N_{2}-1$.

As in the earlier two cases, the row-column decomposition algorithm is executed in two phases. During the first phase the hexagonal array computes a matrix of partial sums $G$ by performing a one-dimensional DFT with respect to index $n_{2}$. During the second phase, a one-dimensional DFT is performed on $G$ with respect to index $n_{1}$. Matrix $y$ is then the desired two-dimensional DFT of the input sequence $x$.

As for the time delays between the entries in the input and output sequences, consider the loop shown in Figure 7(a). Given the orientations of the edges in the loop, we can write $d_{1}+d_{2}+d_{3}-d_{4}=$ 0 . If we assume that the entries of the input, output, and weight sequences all move at the same speed between the processors, we can let $d_{1}=d_{2}=d_{3}=1$ which then implies $d_{4}=3$. It follows that the computations consecutively scheduled on each processor must be executed with three delay units in between. This accounts for the delays between the entries of the input and output sequences as shown in Figure 8. It can further be shown that the first phase of the algorithm needs $3\left(N_{1}-1\right)+1$ steps for the last entry in the $G$ sequence, that is, $G\left(N_{1}-1, N_{2}-1\right)$ to enter the array, and $N_{1}+N_{2}-1$ steps thereafter to move out along the main diagonal which adds up to $4 N_{1}+N_{2}-3$ steps. Similarly, the second phase takes $4 N_{2}+N_{1}-3$ steps, and hence the total number of steps is

$$
T_{\text {hexagonal }}=5\left(N_{1}+N_{2}\right)-6,
$$

and the speedup over the single processor implementation is

$$
S_{\text {hexagonal }}=\frac{N_{1} N_{2}\left(N_{1}+N_{2}\right)}{5\left(N_{1}+N_{2}\right)-6} .
$$

If $N_{1}=N_{2}=N$, then

$$
T_{\text {hexagonal }}=10 N-6
$$

steps overall, and

$$
S_{\text {hexagonal }}=\frac{2 N^{3}}{10 N-6}=O\left(N^{2}\right) .
$$

Even though the speedup in this case is worse than the speedup for the rectangular array in exact terms, it is still asymptotically optimal since $O\left(N^{2}\right)$ processors are used. In fact, one can reduce the constant multiplying $N$ in Equation 17 by using other hexagonal array contractions of the row-column decomposition algorithm. However, this does not change the asymptotic complexity of such contractions which is $O(N)$.

\section{Alignment of Intermediate Results}

In the last section, we described three processor arrays for the row-column decomposition algorithm without discussing how to align the intermediate results between the two phases of 


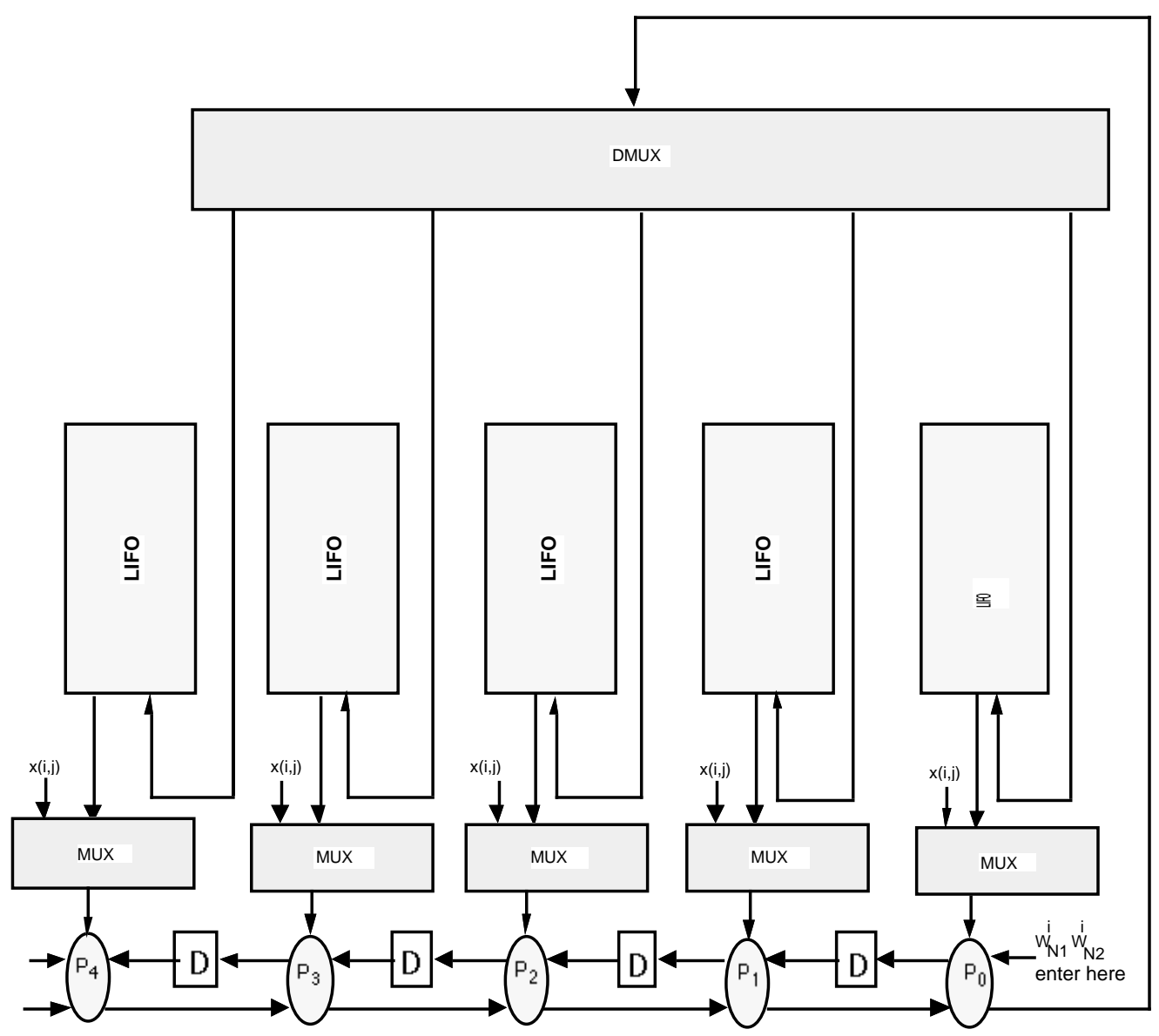

Figure 9. Memory organization for the linear array implementation.

the algorithm. In most array processors, the data between successive phases of an algorithm are aligned by an interconnection network placed between the system's memory and processors [Lawrie 1975, Stone 1971]. However, the three systolic arrays described above do not need such a network, and as we discuss below, a simple LIFO memory is sufficient for aligning the data between the two phases of the row-column decomposition algorithm.

First, consider the linear array implementation and, for simplicity of discussion, let $N_{1}=N_{2}=N$. At the end of the first phase, the $G$ sequence leaves the array as a single stream of outputs which should be organized into a $(2 N-1) \times N$ matrix as it enters the array in the next phase. Notice that the elements with distinct indices in each column are computed in reverse order in the first phase and hence must be realigned for the second phase. A total of $2 N-1$ LIFO memories, each with $N$ locations, can provide the desired alignment of the $G$ sequence (see Figure 9 ). It is possible to save some memory space by observing that not all processors need the same number 


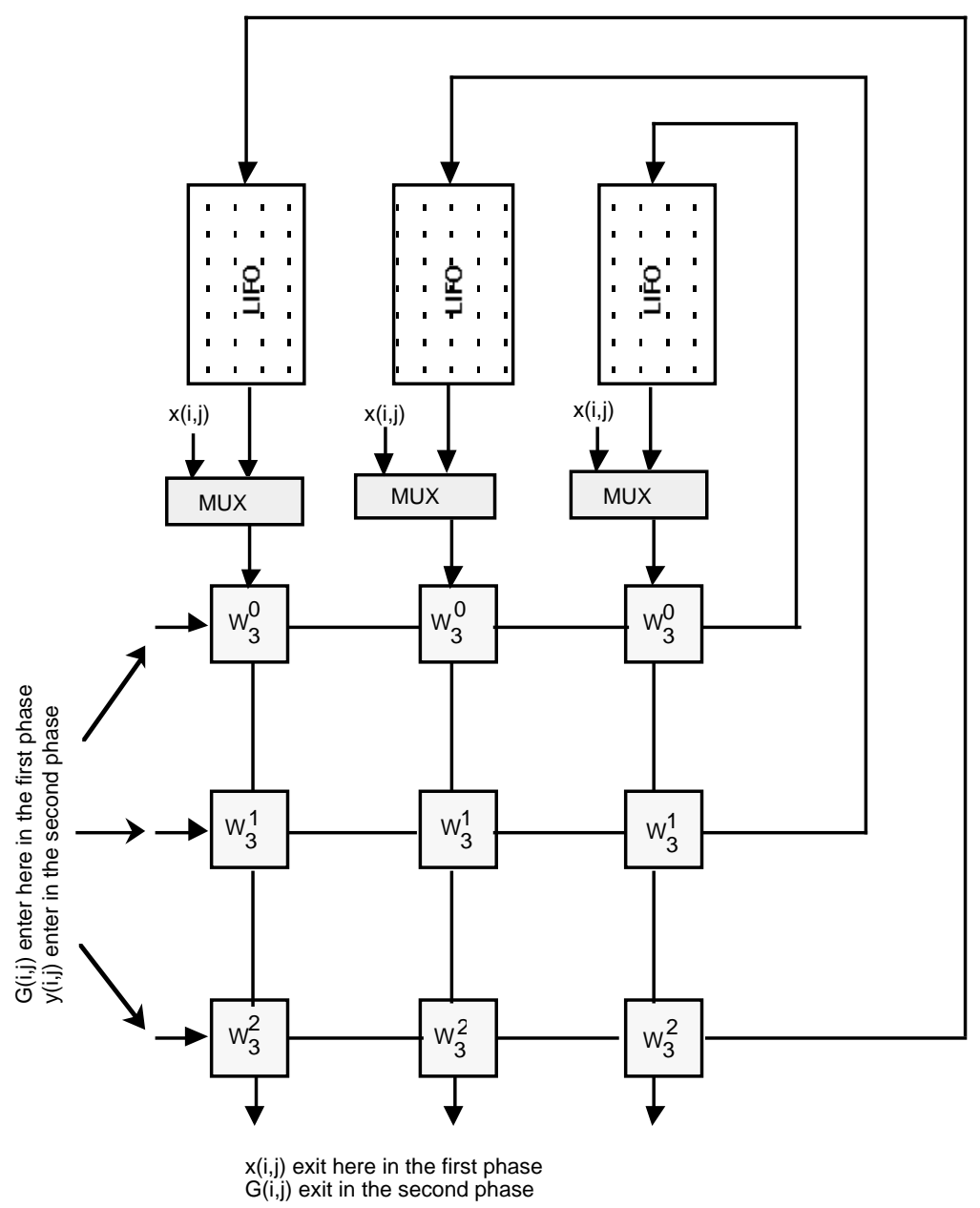

Figure 10. Memory organization for the rectangular array implementation.

of inputs, but this does not change the overall storage complexity of the solution, which is $O\left(N^{2}\right)$. In addition, a demultiplexer is needed to select the right LIFO unit in storing the entries of $G$. The control circuitry for moving the intermediate results back to the array through the LIFOs is a simple exercise in sequential circuit theory and will not be presented here. It suffices to observe that the data which leaves the array in the first phase is needed in the reverse order in the second phase. To select between the $x$ and $G$ sequences for the vertical inputs in the two phases, we can connect a multiplexer circuit to each vertical input of the array and multiplex these two sequences as shown in Figure 9.

Next, consider the rectangular array implementation. This time the intermediate results become available in parallel and hence can be stored simultaneously without using a demultiplexer circuit 
as shown in Figure 10. However, note that the elements of $G$ leave the array in the first phase in reverse order as in the linear array case, and hence LIFO memories are needed in this case as well. This time, however, we need only $N$ LIFOs each with $N$ locations.

Finally, for the hexagonal array, Figure 8 reveals that, as in the first two cases, the $G$ sequence computed in the first phase must be reversed before it enters the array for the second phase. It can be seen that a total of $2 N-1$ LIFOs, each with $N$ locations, is sufficient to carry out this alignment. Like the rectangular array, no demultiplexer is required since the results become available simultaneously. It follows that the total storage complexity of this implementation is the same as that for the linear array.

\section{Extension of Results to Other Arrays and Higher Dimensions}

So far we have discussed the processor array implementations of one and two dimensional DFTs. It should be obvious that the mapping technique is not limited to these dimensions since the contraction of the program graph and task scheduling are carried out for each phase separately, and will be identical for all phases except that the inputs will be different. Thus for an $M$ dimensional DFT, we use the same program graph and the same array -be it linear, rectangular, hexagonal, or any other- with the same timing. The only part which is involved is the alignment of intermediate results between the $M$ phases of the algorithm. The key observation is that we now have $M$ summations whose operands must be multiplexed and permuted into the inputs of a single target array. [Gertner and Shamash 1987] described a general scheme to align the intermediate results by a rotation network which essentially transposes the intermediate results between two consecutive phases. In our designs, we need not use such a network since the transposition, or reversal of the outputs from one phase to the next phase, can be handled with a minor modification of the LIFO memories used in the two-dimensional DFT scheme described above. Rather than have a single LIFO for each vertical input, we now use a pair of LIFOs, between which we alternate the data reversals and feeds. During a given phase, one will be filled with the outputs from the array needed for the next phase, while the other feeds the vertical input to which it is connected with the desired sequence of elements. In the next phase, the roles of the two LIFOs are interchanged so that the one that was filled during the last phase will now feed the data for the vertical input, and the other will be filled with the outputs needed for the following phase. Obviously, this modification does not alter the overall order of complexity of the alignment hardware, and the bounds which hold for the two-dimensional DFT case remain intact.

As for other processor array implementations of the row-column decomposition scheme, it should be obvious from the examples given in Section 4 that one can contract the program graphs in Figure 3 to many other processor arrays by defining other partitions of computation vertices. 
We mentioned a few in Section 4, and many more arrays can be obtained. In general, we can view the computation vertices of the corresponding program graph as a set $S$ of all points in a three-dimensional finite vector space, i.e., $S=\{(x, y, z): x, y, z \epsilon\{0,1, \ldots, N-1\}\}$ which contains $N^{3}$ points. With this representation, any contraction of the program graph can then be viewed as a mapping of $S$ into a set $P$ of processors where $|P|=|S|$. For example, if $P=\left\{p_{1}: 0 \leq i \leq N^{3}\right\}$, then $f: S \rightarrow P$ defined by $f(x, y, z)=p_{z}$ projects all computation vertices on the $z=0$ plane to $p_{0}$, those on the $z=1$ plane to $p_{1}$, and so on. This projection describes a contraction of the original program graph to a linear array with $N$ processors. Other processor arrays can be defined similarly by using other maps from $S$ to $P$. The total number of such maps can easily be shown to be $N^{3 N^{3}}$ for a two-dimensional DFT, and more generally, $N^{(M+1) N^{M+1}}$ for an $M$-dimensional DFT. It can also be shown that not all of these maps lead to systolic arrays, and characterization of those which result in systolic arrays remains an interesting open problem.

\section{Concluding Remarks}

We have examined systolic contractions of multidimensional discrete Fourier transforms with a particular focus on two-dimensional DFTs. It is established that for an M-dimensional DFT, any such contraction can be represented by a mapping of an $(M+1)$-dimensional finite vector space into itself. In more specific terms, three such contractions leading to linear, rectangular, and hexagonal processor arrays have been described. Each of these achieves asymptotically optimal speed up over a single processor implementation, even though the linear array takes $O\left(N^{2}\right)$ time with $O(N)$ processors, and the other two take $O(N)$ time with $O\left(N^{2}\right)$ processors. It should be noted that these time complexities can be reduced to meet the $A T^{2}$ lower bound given in [9]. Nonetheless, this requires a butterfly network which is very costly to lay out in most circuit technologies including VLSI. Thus, the systolic implementations of multidimensional DFT described in this paper may provide a good compromise between speed and cost.

There remain several questions which are worth exploring. We have given only three sytolic implementations of DFTs, and briefly mentioned about a few other possibilities. There are many more processor arrays which can be synthesized from the program graph of the row-column decompisition algorithm using the mapping technique given in the paper. In particular, it will be interesting to determine if there exists a systolic contraction of the row column decomposition algorithm which takes $O(\log N)$ steps for a 2-dimensional sequence of size $N \times N$. Another direction to take is to study other transforms in the context of our mapping procedure. The DFT typifies a wide range of discrete transforms, but there are other discrete transformations such as binomial sums which should be examined separately. Finally, it will be worthwhile to examine systolic contractions of the fast Fourier fransform (FFT) algorithm. Only a few efforts have been reported 
recently on parallel FFT schemes, and a lot needs to be done to reveal the tradeoffs available between cost and time in various implementations.

\section{ACKNOWLEDGMENTS}

The authors thank the referees for their constructive comments and criticisms. They are also grateful to the Managing Editor for her editorial suggestions. 


\section{References}

[1] Blahut, R.E. 1985. Fast Algorithms for Digital Signal Processing, Addison-Wesley, Reading, MA.

[2] Capello P. and Steiglitz K. 1983. Unifying VLSI array designs with geometric transformations. In Conference Proceedings-International Conference on Parallel Processing (St. Charles, IL, Aug.), pp. 448-457.

[3] Chakrabarti C. and Ja'Ja' J. 1988. Optimal Architectures for multidimensional transforms. Computer Science Technical Report- UMIACS-TR-88-36, CS-TR-2031 (University of Maryland, College Park, MD, May).

[4] Chowdary N.U. 1984. A high speed two dimensional FFT processor. In Conference Proceedings-ICASSP (San Diego, CA, Mar.), pp. 4.11.1-4.11.4, San Diego, CA, Mar. 1984.

[5] Cytron R. 1986. Doacross: Beyond vectorization for multiprocessors (Extended Abstract). In Conference Proceedings-International Conference on Parallel Processing (St. Charles, IL, Aug.), pp. 836-844.

[6] Dudgeon D.E. and Mersereau R. M. 1984. Multidimensional Digital Signal Processing, Prentice-Hall, Englewood Cliffs, NJ.

[7] Jacobson L. and Wechsler H. 1984. A theory for invariant object recognition in the front of parallel plane. IEEE Transactions on Pattern Analysis and Machine Intelligence, Vol. PAMI-6 (May), 325-331.

[8] Franklin M.A. 1981. VLSI performance of banyan and crossbar communication networks. IEEE Transactions on Computers, Vol. C-30, No. 4 (Apr.), 283-290.

[9] Gafni H. and Zeevi Y.Y. 1979. A model for processing of movement in the visual system. Biology and Cybernetics, Vol. 32, 165-173.

[10] Gertner I. and Shamash M. 1987. VLSI architectures for multidimensional Fourier transform processing. IEEE Transactions on Computers, Vol. C-36, No. 11 (Nov.), pp. 1265-1274.

[11] Hinshaw W S. and Lent A.H. 1983. An introduction to NMR imaging: From the block equation to the imaging equation Proc. IEEE, Vol. 71, No. 3 (Mar.)

[12] Lawrie D.H. 1975. Access and alignment of data in an array processor. IEEE Transactions on Computers, Vol. C-24 (Dec.), 1145-1155.

[13] Leiserson C.E. 1981. Area-efficient VLSI computation, The MIT Press, Cambridge, MA.

[14] Ling N.and Bayoumi M. 1988. Algorithms for high speed multidimensional arithmetic and DSP systolic arrays. In Conference Proceedings-International Conference on Parallel Processing (St. Charles, IL, Aug.), pp. 367-374.

[15] Kung H.T. and Leiserson C.E. 1980. Algorithms for VLSI processor arrays. in Introduction to VLSI systems, Mead and Conway, Eds. Reading, MA: Addison-Wesley. 
[16] Miranker W.L. and Winkler A. 1984. Spacetime representation of computational structures. Computing, Vol. C-32, No. 2, 93-114.

[17] Oppenheim A. and Schafer R. 1975. Digital Signal Processing, Prentice-Hall, Englewood Cliffs, NJ.

[18] Ramakrishnan I.V., Fussel D.S. and Silberchartz A. 1986. Mapping homogeneous graphs on linear arrays. IEEE Transactions Computers, Vol. C-35 No. 3 (Mar.),189-209.

[19] Ramakrishnan I.V. and Varman P.J. 1984. On mapping cube graphs on VLSI arrays. In Proceedings- Fourth International Conference on Foundations of Software Technology, Lecture Notes in Computer Science, Vol. 181, Springer-Verlag, Berlin.

[20] Shen W. 1987. On mapping algorithms onto processor arrays. Ph.D. Disser. (Dec.) ECSE Department, RPI.

[21] Shen W. and Oruç A.Y. 1989. On systolic contractions of program graphs. IEEE Transactions on Computers, Vol. C-38. No. 10 (Oct.) 1451-1457.

[22] Stone H.S. 1971. Parallel processing with the perfect shuffle. IEEE Transactions Computers, Vol. C-20, No.2 (Feb.), 153-161.

[23] Thompson C.D. 1983. Fourier transforms in VLSI. IEEE Transactions Computers, Vol. C-32, No. 11 (Nov.), 1047-1057.

[24] Zhang C.N. and Yun Y.Y. 1984. Multidimensional systolic networks for discrete Fourier transform. In Conference Proceedings- 11th Annual International Symposium on Computer Architecture (Ann Arbor, MI.) pp. 215-222. 